DEMONSTRATIO MATHEMATICA

Vol. XLIII No $2 \quad 2010$

\author{
Masaki Kasedou*
}

\title{
SPACELIKE SUBMANIFOLDS IN DE SITTER SPACE
}

\begin{abstract}
We investigate the differential geometry of spacelike submanifolds of codimension at least two in de Sitter space as an application of the theory of Legendrian singularities. We also discuss related geometric property of spacelike hypersurfaces in de Sitter space.
\end{abstract}

\section{Introduction}

It is known that de Sitter space is a Lorentzian space form with a positive curvature. Recently, Izumiya, Pei and Sano [3] investigated the extrinsic differential geometry of hypersurfaces in the hyperbolic space by applying the theory of Legendrian singularities. The main tool is a lightcone Gauss indicatrix, which is defined by a lightlike normal of hypersurface, and their singularity sets correspond to lightcone parabolic sets of hypersurfaces. For higher codimension case, the normal vector is not uniquely determined, however it is possible to construct hypersurfaces from normal unit vector fields of the subspace. Izumiya, Pei, Romero Fuster and Takahashi [6] introduced the notion of canal hypersurfaces and horospherical hypersurfaces from the normal frames of submanifolds in the hyperbolic space, and investigated submanifolds of higher codimension in the hyperbolic space from the viewpoint of singularity theory. On the other hand, the differential geometry of de Sitter space is also studied. In [7] we introduced the notion of lightcone Gauss image which is an analogous tool introduced in [3], and investigate the case of spacelike hypersurface in de Sitter space. For codimension two case, Fusho and Izumiya [2] firstly introduced the notion of lightlike surface of a spacelike curve in the de Sitter three-space. In [8] we investigated singularities of lightlike hypersurface of spacelike submanifold of codimension

2000 Mathematics Subject Classification: 53A35, 53B30, 58C25.

Key words and phrases: de Sitter space, spacelike submanifold, spacelike hypersurface.

${ }^{*}$ This work was supported by the JSPS International Training Program(ITP). 
two in de Sitter $n$-space for $n \geq 3$ by using the lightlike normal direction, which is an analogous study in the Minkowski space $[4,5]$.

In this paper, we argue an analogous study of the submanifolds of higher codimension in hyperbolic space [6] and introduce the notions of horospherical hypersurfaces and spacelike canal hypersurfaces by using timelike unit normal vector fields. The singular point of horospherical surface corresponds to the parabolic point of spacelike canal hypersurface, which we call a horospherical point, and the spacelike submanifold is tangent to a de Sitter hyperhorosphere at the horospherical point. If we assume a hypothesis of Theorem 6.5 , then a contact type of a de Sitter hyperhorosphere and a spacelike submanifold corresponds to a singular type of horospherical hypersurface, and also corresponds to a singular type of lightcone Gauss image of spacelike canal surface. In this paper we consider timelike normal direction of spacelike submanifolds, so that this study is not a generalization of $[8,9]$. In $\S 2$ we review briefly the basic notions of differential geometry of spacelike hypersurfaces [7]. In $\S 3,4$ we define a timelike normal vector field of spacelike submanifolds in de Sitter space and introduce a notion of horospherical height function and horospherical hypersurface. We also define a spacelike canal hypersurface, whose lightcone Gauss image is diffeomorphic to a horospherical hypersurface. In $\S 5$ we naturally interpret a horospherical hypersurfaces of a spacelike submanifold as a wave front set of horospherical height functions in the theory of Legendrian singularities. In $\S 6$ we use the theory of contacts between the submanifolds due to Montaldi [10], and we discuss geometric properties of singularities of horospherical hypersurfaces. We also consider generic properties of spacelike submanifolds.

\section{Spacelike hypersurfaces in de Sitter space}

In this section we review the extrinsic differential geometry of spacelike hypersurfaces in de Sitter space [7], which is an analogous study of [3]. Let $\mathbb{R}^{n+1}=\left\{\mathbf{x}=\left(x_{0}, \ldots, x_{n}\right) \mid x_{i} \in \mathbb{R}(i=0, \ldots, n)\right\}$ be an $(n+1)$-dimensional vector space. For any vectors $\mathbf{x}=\left(x_{0}, \ldots, x_{n}\right), \mathbf{y}=\left(y_{0}, \ldots, y_{n}\right)$ in $\mathbb{R}^{n+1}$, the pseudo scalar product of $\mathbf{x}$ and $\mathbf{y}$ is defined by $\langle\mathbf{x}, \mathbf{y}\rangle=-x_{0} y_{0}+\sum_{i=1}^{n} x_{i} y_{i}$. We call $\left(\mathbb{R}^{n+1},\langle\rangle,\right)$ a Minkowski $(n+1)$-space and write $\mathbb{R}_{1}^{n+1}$ instead of $\left(\mathbb{R}^{n+1},\langle\rangle,\right)$.

We say that a vector $\mathbf{x} \in \mathbb{R}_{1}^{n+1} \backslash\{\mathbf{0}\}$ is spacelike, lightlike or timelike if $\langle\mathbf{x}, \mathbf{x}\rangle>0,\langle\mathbf{x}, \mathbf{x}\rangle=0$ or $\langle\mathbf{x}, \mathbf{x}\rangle\langle 0$ respectively. The norm of the vector $\mathbf{x} \in \mathbb{R}_{1}^{n+1}$ is defined by $\|\mathbf{x}\|=\sqrt{|\langle\mathbf{x}, \mathbf{x}\rangle|}$. For a vector $\mathbf{v} \in \mathbb{R}_{1}^{n+1} \backslash\{\mathbf{0}\}$ and a real number $c$, we define a hyperplane with pseudo normal $\mathbf{v}$ in the Minkowski space by $\operatorname{HP}(\mathbf{v}, c)=\left\{\mathbf{x} \in \mathbb{R}_{1}^{n+1} \mid\langle\mathbf{x}, \mathbf{v}\rangle=c\right\}$. We say that a hyperplane $H P(\mathbf{v}, c)$ is spacelike, timelike or lightlike if the vector $\mathbf{v}$ is timelike, spacelike or lightlike. 
We now respectively define hyperbolic $n$-space and de Sitter $n$-space by

$$
\begin{aligned}
H_{ \pm}^{n}(-1) & =\left\{\mathbf{x} \in \mathbb{R}_{1}^{n+1} \mid\langle\mathbf{x}, \mathbf{x}\rangle=-1, \operatorname{sgn}\left(x_{0}\right)= \pm 1\right\}, \\
S_{1}^{n} & =\left\{\mathbf{x} \in \mathbb{R}_{1}^{n+1} \mid\langle\mathbf{x}, \mathbf{x}\rangle=1\right\},
\end{aligned}
$$

and we write $H^{n}(-1)=H_{+}^{n}(-1) \cup H_{-}^{n}(-1)$. For any $\mathbf{x}_{1}, \mathbf{x}_{2}, \ldots, \mathbf{x}_{n} \in \mathbb{R}_{1}^{n+1}$, we can define a vector $\mathbf{x}_{1} \wedge \mathbf{x}_{2} \wedge \ldots \wedge \mathbf{x}_{n}$ with the property $\left\langle\mathbf{x}, \mathbf{x}_{1} \wedge \ldots \wedge \mathbf{x}_{n}\right\rangle=$ $\operatorname{det}\left(\mathbf{x}, \mathbf{x}_{1}, \ldots, \mathbf{x}_{n}\right)$, so that $\mathbf{x}_{1} \wedge \ldots \wedge \mathbf{x}_{n}$ is pseudo-orthogonal to any $\mathbf{x}_{i}$ for $i=1, \ldots, n$. We also define future (resp. past) lightcone at the origin by

$$
\begin{aligned}
& L C_{+}^{*}=\left\{\mathbf{x} \in \mathbb{R}_{1}^{n+1} \mid\langle\mathbf{x}, \mathbf{x}\rangle=0, x_{0}>0\right\}, \\
& L C_{-}^{*}=\left\{\mathbf{x} \in \mathbb{R}_{1}^{n+1} \mid\langle\mathbf{x}, \mathbf{x}\rangle=0, x_{0}<0\right\},
\end{aligned}
$$

and we write $L C^{*}=L C_{+}^{*} \cap L C_{-}^{*}$.

Let $\mathbf{X}: U \rightarrow S_{1}^{n}$ be an embedding, where $U \subset \mathbb{R}^{n-1}$ is an open subset. We say $\mathbf{X}$ is a spacelike hypersurface in $S_{1}^{n}$ if every non zero vector generated by $\left\{\mathbf{X}_{u_{i}}(\mathbf{u})\right\}_{i=1}^{n-1}$ is always spacelike, where $\mathbf{u}=\left(u_{1}, \ldots, u_{n-1}\right)$ is an element of $U$ and $\mathbf{X}_{u_{i}}$ is a partial derivative of $\mathbf{X}$ with respect to $u_{i}$. We denote $M=$ $\mathbf{X}(U)$ and identify $M$ with $U$ through the embedding $\mathbf{X}$. Since $\langle\mathbf{X}, \mathbf{X}\rangle \equiv 1$, we have $\left\langle\mathbf{X}_{u_{i}}, \mathbf{X}\right\rangle \equiv 0$ for $i=1, \ldots, n-1$. It follows that a hyperplane spanned by $\left\{\mathbf{X}, \mathbf{X}_{u_{1}}, \ldots, \mathbf{X}_{u_{n-1}}\right\}$ is spacelike. We define a vector $\mathbf{e}(\mathbf{u})=$ $\mathbf{X}(\mathbf{u}) \wedge \mathbf{X}_{u_{1}}(\mathbf{u}) \wedge \ldots \wedge \mathbf{X}_{u_{n-1}}(\mathbf{u}) /\left\|\mathbf{X}(\mathbf{u}) \wedge \mathbf{X}_{u_{1}}(\mathbf{u}) \wedge \ldots \wedge \mathbf{X}_{u_{n-1}}(\mathbf{u})\right\|$. Then $\mathbf{e}$ is pseudo orthogonal to $\mathbf{X}$ and $\mathbf{X}_{u_{i}}$ for $i=1, \ldots, n-1$. We define a map $\mathbb{L}^{ \pm}: U \rightarrow L C_{ \pm}^{*}$ by

$$
\mathbb{L}^{ \pm}(\mathbf{u})=\mathbf{X}(\mathbf{u}) \pm \mathbf{e}(\mathbf{u})
$$

which is called a positive (resp. negative) lightcone Gauss image of $\mathbf{X}$.

We now consider a hypersurface defined by $H P(\mathbf{v}, c) \cap S_{1}^{n}$. We say that $H P(\mathbf{v}, c) \cap S_{1}^{n}$ is an elliptic hyperquadric or a hyperbolic hyperquadric if $H P(\mathbf{v}, c)$ is spacelike or timelike respectively. We say that $H P(\mathbf{v}, c) \cap S_{1}^{n}$ is a de Sitter hyperhorosphere if $c \neq 0$ and $H P(\mathbf{v}, c)$ is lightlike. We have the following proposition analogous to ([3], Proposition 2.2).

Proposition 2.1. ([7]) Let $\mathbf{X}: U \rightarrow S_{1}^{n}$ be a spacelike hypersurface in $S_{1}^{n}$. The lightcone Gauss image $\mathbb{L}^{ \pm}$is constant if and only if the spacelike hypersurface $M=\mathbf{X}(U)$ is a part of a de Sitter hyperhorosphere.

We now define the lightcone Gauss-Kronecker curvature and the lightcone mean curvature of the spacelike hypersurface $M=\mathbf{X}(U)$. For any $p \in M$ and $\mathbf{v} \in T_{p} M$, we can show $D_{\mathbf{v}} \mathbf{e}$ and $D_{\mathbf{v}} \mathbb{L}^{ \pm} \in T_{p} M$, where $D_{\mathbf{v}}$ is the covariant derivative with respect to the tangent vector $\mathbf{v}$. Under the identification of $U$ and $M, d \mathbb{L}^{ \pm}(\mathbf{u})$ is a linear transformation on $T_{p} M$. We call $S_{p}^{ \pm}=-d \mathbb{L}^{ \pm}(\mathbf{u})$ a lightcone shape operator of $M=\mathbf{X}(U)$ of at $p=\mathbf{X}(\mathbf{u})$. 
The lightcone Gauss-Kronecker curvature $K_{\ell}^{ \pm}$of $M=\mathbf{X}(U)$ at $p=\mathbf{X}(\mathbf{u})$ is defined to be the determinant of the lightcone shape operator.

Since $\mathbf{X}_{u_{i}}$ (for $i=1, \ldots, n-1$ ) are spacelike vectors, we have the Riemannian metric (first fundamental form) $d s^{2}=\sum_{i, j=1}^{n-1} g_{i j} d u_{i} d u_{j}$ on $M=\mathbf{X}(U)$, where $g_{i j}(\mathbf{u})=\left\langle\mathbf{X}_{u_{i}}(\mathbf{u}), \mathbf{X}_{u_{j}}(\mathbf{u})\right\rangle$ for any $\mathbf{u} \in U$. We define a lightcone second fundamental invariants by $\bar{h}_{i j}^{ \pm}(\mathbf{u})=\left\langle-\mathbb{L}_{u_{i}}^{ \pm}(\mathbf{u}), \mathbf{X}_{u_{j}}(\mathbf{u})\right\rangle$ for any $\mathbf{u} \in U$. In [7] we obtained explicit expression for the lightcone Gauss-Kronecker curvature:

$$
K_{\ell}^{ \pm}=\operatorname{det}\left(\bar{h}_{i j}^{ \pm}\right) / \operatorname{det}\left(g_{\alpha \beta}\right) \text {. }
$$

We say that $p=\mathbf{X}(\mathbf{u})$ is a lightcone parabolic point of $\mathbf{X}$ if $K_{\ell}^{ \pm}(\mathbf{u})=0$.

We define a family of functions $H: U \times L C^{*} \rightarrow \mathbb{R}$ by

$$
H(\mathbf{u}, \mathbf{v})=\langle\mathbf{X}(\mathbf{u}), \mathbf{v}\rangle-1
$$

which we call a lightcone height function of $M$. We have the following proposition analogous to ([3], Proposition 3.1).

Proposition 2.2. ([7]) Let $H$ be a lightcone height function, then $H(\mathbf{u}, \mathbf{v})$ $=\partial H(\mathbf{u}, \mathbf{v}) / \partial u_{i}=0$ for $i=1, \ldots, n-1$ if and only if $\mathbf{v}=\mathbb{L}^{ \pm}(\mathbf{u})$.

We also naturally interpreted the lightcone Gauss image of a spacelike hypersurface as a wave front set in the frame work of contact geometry in [7]. This is the analogous way to the differential geometry of hypersurfaces in hyperbolic space [3].

Let $\pi^{ \pm}: P T^{*}\left(L C_{ \pm}^{*}\right) \rightarrow L C_{ \pm}^{*}$ be the projective cotangent bundles with canonical contact structures. Consider the tangent bundle $\tau^{ \pm}: T P T^{*}\left(L C_{ \pm}^{*}\right)$ $\rightarrow P T^{*}\left(L C_{ \pm}^{*}\right)$ and the differential map $d \pi^{ \pm}: T P T^{*}\left(L C_{ \pm}^{*}\right) \rightarrow T\left(L C_{ \pm}^{*}\right)$ of $\pi^{ \pm}$. For any $X \in T P T^{*}\left(L C_{ \pm}^{*}\right)$, there exists an element $\alpha \in T^{*}\left(L C_{ \pm}^{*}\right)$ such that $\tau^{ \pm}(X)=[\alpha]$. For $\mathbf{v} \in L C_{ \pm}^{*}$ and $V \in T_{\mathbf{v}}\left(L C_{ \pm}^{*}\right)$, the property $\alpha(V)=0$ does not depend on the choice of representative of the class $[\alpha]$. Thus, we can define the canonical contact structure on $P T^{*}\left(L C_{ \pm}^{*}\right)$ by

$$
K^{ \pm}=\left\{X \in T P T^{*}\left(L C_{ \pm}^{*}\right) \mid \tau^{ \pm}(X)\left(d \pi^{ \pm}(X)\right)=0\right\} .
$$

On the other hand, we consider a point $\mathbf{v}=\left(v_{0}, v_{1}, \ldots, v_{n}\right) \in L C_{ \pm}^{*}$, then we have the relation $v_{0}= \pm \sqrt{v_{1}^{2}+\cdots+v_{n}^{2}}$. So we adopt the coordinate system $\left(v_{1}, \ldots, v_{n}\right)$ of the manifold $L C_{ \pm}^{*}$. Then we have the trivialization $P T^{*}\left(L C_{ \pm}^{*}\right) \equiv L C_{ \pm}^{*} \times P^{*} \mathbb{R}^{n-1}$, and call $\left(\left(v_{0}, \ldots, v_{n}\right),\left[\xi_{1}: \ldots: \xi_{n}\right]\right)$ homogeneous coordinates of $P T^{*}\left(L C_{ \pm}^{*}\right)$, where $\left[\xi_{1}: \ldots: \xi_{n}\right]$ are the homogeneous coordinates of the dual projective space $P^{*} \mathbb{R}^{n-1}$.

It is easy to show that $X_{\bullet} \in K_{\bullet}^{ \pm}$if and only if $\sum_{i=1}^{n} \mu_{i} \xi_{i}=0$, where - $=(\mathbf{v},[\xi])$ and $d \pi_{\bullet}^{ \pm}\left(X_{\bullet}\right)=\sum_{i=1}^{n} \mu_{i} \partial / \partial v_{i} \in T_{\bullet} L C_{ \pm}^{*}$. An immersion $i$ : $L \rightarrow P T^{*}\left(L C_{ \pm}^{*}\right)$ is said to be a Legendrian immersion if $\operatorname{dim} L=n-1$ and $d i_{q}\left(T_{q} L\right) \subset K_{i(q)}$ for any $q \in L$. The map $\pi \circ i$ is also called the Legendrian 
map and the image $W(i)=$ image $(\pi \circ i)$, the wave front of $i$. Moreover, $i$ (or the image of $i$ ) is called the Legendrian lift of $W(i)$.

Let $F:\left(\mathbb{R}^{s} \times \mathbb{R}^{k},\left(\mathbf{u}_{0}, \mathbf{v}_{0}\right)\right) \rightarrow(\mathbb{R}, 0)$ be a function germ. We say that $F$ is a Morse family of hypersurfaces if the map germ $\Delta^{*} F:\left(\mathbb{R}^{s} \times \mathbb{R}^{k},\left(\mathbf{u}_{0}, \mathbf{v}_{0}\right)\right) \rightarrow$ $\left(\mathbb{R}^{s+1}, \mathbf{0}\right)$ defined by

$$
\Delta^{*} F=\left(F, \frac{\partial F}{\partial u_{1}}, \ldots, \frac{\partial F}{\partial u_{s}}\right)
$$

is non singular. In this case, we have a smooth $(k-1)$-dimensional smooth submanifold,

$$
\begin{aligned}
\Sigma_{*}(F)=\left\{(\mathbf{u}, \mathbf{v}) \in\left(\mathbb{R}^{n-r} \times \mathbb{R}^{k},\left(\mathbf{u}_{0}, \mathbf{v}_{0}\right)\right)\right. & \\
& \left.F(\mathbf{u}, \mathbf{v})=\frac{\partial F}{\partial u_{1}}(\mathbf{u}, \mathbf{v})=\ldots=\frac{\partial F}{\partial u_{n-r}}(\mathbf{u}, \mathbf{v})=0\right\},
\end{aligned}
$$

and the map germ $\mathcal{L}_{F}:\left(\Sigma_{*}(F),\left(\mathbf{u}_{0}, \mathbf{v}_{0}\right)\right) \rightarrow P T^{*} \mathbb{R}^{k}$ defined by

$$
\mathcal{L}_{F}(\mathbf{u}, \mathbf{v})=\left(\mathbf{v},\left[\frac{\partial F}{\partial v_{1}}(\mathbf{u}, \mathbf{v}): \ldots: \frac{\partial F}{\partial v_{k}}(\mathbf{u}, \mathbf{v})\right]\right)
$$

is a Legendrian immersion germ. Then we have the following fundamental theorem of Arnol'd and Zakalyukin [1, 12].

Proposition 2.3. All Legendrian submanifold germs in $P T^{*} \mathbb{R}^{k}$ are constructed by the above method.

We call $F$ a generating family of $\mathcal{L}_{F}\left(\Sigma_{*}(F)\right)$. Therefore the wave front is

$$
\begin{aligned}
W\left(\mathcal{L}_{F}\right)= & \left\{\mathbf{v} \in \mathbb{R}^{k} \mid \exists \mathbf{u} \in \mathbb{R}^{n-r}\right. \\
& \text { such that } \left.F(\mathbf{u}, \mathbf{v})=\frac{\partial F}{\partial u_{1}}(\mathbf{u}, \mathbf{v})=\cdots=\frac{\partial F}{\partial u_{n-r}}(\mathbf{u}, \mathbf{v})=0\right\} .
\end{aligned}
$$

We call it the discriminant set of $F$. In [9] we showed that the lightcone height function $H$ is a Morse family of hypersurface and its discriminant set is the image of lightcone Gauss images $\mathbb{L}^{ \pm}(U)$. Therefore we have a immersion germ $\mathcal{L}^{ \pm}:\left(\Sigma_{*}^{ \pm}(H),\left(\mathbf{u}_{0}, \mathbf{v}_{0}^{ \pm}\right)\right) \rightarrow P T^{*}\left(L C_{ \pm}^{*}\right)$ defined by

$$
\mathcal{L}^{ \pm}(\mathbf{u})=\left(\mathbf{v}^{ \pm},\left[\frac{\partial H}{\partial v_{1}}\left(\mathbf{u}, \mathbf{v}^{ \pm}\right): \ldots: \frac{\partial H}{\partial v_{n}}\left(\mathbf{u}, \mathbf{v}^{ \pm}\right)\right]\right)
$$

where $\mathbf{v}^{ \pm}=\mathbb{L}^{ \pm}(\mathbf{u})$ and $\Sigma_{*}^{ \pm}(H)$ is a singular set of $H$. 


\section{Spacelike submanifolds in de Sitter space}

In this section, we consider the differential geometry of spacelike submanifolds in de Sitter space, which is analogous to [6].

Let $r \geq 2$ be an integer and $\mathbf{X}: U \rightarrow S_{1}^{n}$ be an embedding from an open set $U \subset \mathbb{R}^{n-r}$. We say that $\mathbf{X}$ is spacelike in $S_{1}^{n}$ if every non zero vector generated by $\left\{\mathbf{X}_{u_{i}}(\mathbf{u})\right\}_{i=1}^{n-r}$ is spacelike, where $\mathbf{u} \in U$ and $\mathbf{X}_{u_{i}}=\partial \mathbf{X} / \partial u_{i}$. We identify $M=\mathbf{X}(U)$ with $U$ through the embedding $\mathbf{X}$ and call $M$ a spacelike submanifold of codimension $r$ in de Sitter space. Since $\langle\mathbf{X}, \mathbf{X}\rangle \equiv 1$, so that $\left\langle\mathbf{X}_{u_{i}}, \mathbf{X}\right\rangle \equiv 0$ for $i=1, \ldots, n-r$. The tangent space of $M$ at $p=\mathbf{X}(\mathbf{u})$ is spanned by the vectors $\mathbf{X}_{u_{i}}(\mathbf{u})$ for $i=1, \ldots, n-r$.

Let $N_{p} M$ be the normal space of $M$ at $p$ in $\mathbb{R}_{1}^{n+1}$ and we define $N_{p}^{*}(M)=$ $N_{p} M \cap T_{p} S_{1}^{n}$. Let $\mathbf{n}: U \rightarrow N_{p}^{*}(M)$ be a timelike unit normal vector field on $M$. Since $\langle\mathbf{n}, \mathbf{n}\rangle \equiv-1$ and $\langle\mathbf{X}, \mathbf{n}\rangle \equiv 0, \mathbf{n}_{u_{i}}$ is pseudo orthogonal to both of $\mathbf{X}$ and $\mathbf{n}$ for $i=1, \ldots, n-r$. Therefore we have $\mathbf{n}_{u_{i}}(\mathbf{u}) \in T_{p} M \oplus N_{p}^{*}(M)$. Consider two pseudo orthonormal projections

$$
\pi_{p}^{t}: T_{p} \mathbb{R}_{1}^{n+1} \rightarrow T_{p} M, \quad \pi_{p}^{n}: T_{p} \mathbb{R}_{1}^{n+1} \rightarrow N_{p} M .
$$

Let $d_{\mathbf{u}} \mathbf{n}$ be the derivative of $\mathbf{n}$ at $\mathbf{u}$, under the identification of $M$ and $U$ through $\mathbf{X}$, we have the linear transformations on $T_{p} M$

$$
d_{p} \mathbf{n}^{T}=\pi_{p}^{t} \circ d_{\mathbf{u}} \mathbf{n}, \quad d_{p} \mathbf{n}^{N}=\pi_{p}^{n} \circ d_{\mathbf{u}} \mathbf{n} .
$$

We respectively call the linear transformation $A_{p}(\mathbf{n})=-d_{p} \mathbf{n}^{T}$ and $S_{p}(\mathbf{n})=$ $-\left(\operatorname{id}_{T_{p}} M+d_{p} \mathbf{n}^{T}\right)$ an $\mathbf{n}$-shape operator and a horospherical $\mathbf{n}$-shape operator of $M$ at $p=\mathbf{X}(\mathbf{u})$. We also call the linear map $d_{\mathbf{u}} \mathbf{n}^{N}$ a normal connection with respect to the timelike normal $\mathbf{n}$ of $M$.

We denote eigenvalues of $A_{p}(\mathbf{n})$ and $S_{p}(\mathbf{n})$ by $\kappa_{p}(\mathbf{n})$ and $\bar{\kappa}_{p}(\mathbf{n})$, which we respectively call an $\mathbf{n}$-principal curvature and a horospherical $\mathbf{n}$-principal curvature. The horospherical Gauss-Kronecker curvature with respect to $\mathbf{n}$ at $p=\mathbf{X}(\mathbf{u})$ is defined to be

$$
K_{h}(\mathbf{n})(\mathbf{u})=\operatorname{det} S_{p}(\mathbf{n}) .
$$

We say that a point $p_{0}=\mathbf{X}\left(\mathbf{u}_{0}\right)$ is an $\mathbf{n}$-umbilic point if $S_{p_{0}}(\mathbf{n})=\bar{\kappa}_{p_{0}}(\mathbf{n}) \operatorname{id}_{T_{p_{0}} M}$. Since the eigenvectors of $S_{p_{0}}(\mathbf{n})$ and $A_{p_{0}}(\mathbf{n})$ are the same, the above condition is equivalent to $A_{p_{0}}(\mathbf{n})=\kappa_{p_{0}}(\mathbf{n}) \mathrm{id}_{T_{p_{0}} M}$. We say that the spacelike submanifold $M$ is totally $\mathbf{n}$-umbilic if every point on $M$ is $\mathbf{n}$-umbilic. We also say that the timelike unit normal vector field $\mathbf{n}$ is parallel at $p_{0}$ if $d_{p_{0}} \mathbf{n}^{N}=0_{T_{p_{0}} M}$. The timelike unit normal field $\mathbf{n}$ is parallel if $\mathbf{n}$ is parallel at any points on $M$. Then we have the following result which is analogous to ([6], Proposition 3.1).

Proposition 3.1. Let $\mathbf{X}: U \rightarrow S_{1}^{n}$ be a spacelike submanifold of codimension $r \geq 2$. Suppose that $M=\mathbf{X}(U)$ is totally $\mathbf{n}$-umbilic, where $\mathbf{n}$ is a timelike unit normal parallel vector field. Then $\kappa_{p}(\mathbf{n})$ and $\bar{\kappa}_{p}(\mathbf{n})$ are constant 
$\kappa(\mathbf{n})$ and $\bar{\kappa}(\mathbf{n})$, and there exists a vector $\mathbf{v} \in \mathbb{R}_{1}^{n+1}$ and real number $c$ such that $M$ is a part of a hyperquadric $H P(\mathbf{v}, c) \cap S_{1}^{n}$ in de Sitter space. Under this condition we have following cases:

(1) If $1<|\bar{\kappa}(\mathbf{n})+1|=|\kappa(\mathbf{n})|$ then $M$ is a part of a hyperbolic hyperquadric $H P(\mathbf{v},+1)$.

(2) If $0<|\bar{\kappa}(\mathbf{n})+1|=|\kappa(\mathbf{n})|<1$ then $M$ is a part of an elliptic hyperquadric $H P(\mathbf{v},+1)$.

(3) If $\bar{\kappa}(\mathbf{n})+1=\kappa(\mathbf{n})=0$ then $M$ is a part of an elliptic hyperquadric $H P(\mathbf{v}, 0)$.

(4) If $\kappa(\mathbf{n})=1$ (namely $\bar{\kappa}(\mathbf{n})=0$ ) then $M$ is a part of a de Sitter hyperhorosphere $H P(\mathbf{v},+1)$.

Proof. By the assumption, we have $A_{p}(\mathbf{n}) \equiv \kappa_{p} \mathrm{id}_{T_{p} M}$. This means that $\pi_{p}^{T} \circ \mathbf{n}_{u_{i}}(\mathbf{u}) \equiv \kappa_{p} \mathbf{X}_{u_{i}}(\mathbf{u})$. Since $\mathbf{n}$ is parallel, we have $\mathbf{n}_{u_{i}}(\mathbf{u})=\kappa_{p} \mathbf{X}_{u_{i}}(\mathbf{u})$. So that $\mathbf{n}_{u_{i} u_{j}}(\mathbf{u})=\kappa_{u_{j}, p} \mathbf{X}_{u_{i}}(\mathbf{u})+\kappa_{p} \mathbf{X}_{u_{i} u_{j}}(\mathbf{u})$ and $\mathbf{n}_{u_{j} u_{i}}(\mathbf{u})=\kappa_{u_{i}, p} \mathbf{X}_{u_{j}}(\mathbf{u})+$ $\kappa_{p} \mathbf{X}_{u_{j} u_{i}}(\mathbf{u})$. It follows that $\mathbf{X}_{u_{i} u_{j}} \equiv \mathbf{X}_{u_{j} u_{i}}$ and $\mathbf{n}_{u_{i} u_{j}} \equiv \mathbf{n}_{u_{j} u_{i}}$, then we have $\kappa_{u_{j}, p} \mathbf{X}_{u_{i}}(\mathbf{u})=\kappa_{u_{i}, p} \mathbf{X}_{u_{j}}(\mathbf{u})$. Since $\mathbf{X}_{i}(\mathbf{u})$ and $\mathbf{X}_{j}(\mathbf{u})$ are linearly independent, $\kappa_{u_{i}, p}=\kappa_{u_{j}, p}=0$. This means that $\kappa_{p}$ and $\bar{\kappa}_{p}$ are constant $\kappa$ and $\bar{\kappa}$.

We now assume that $\bar{\kappa}+1=\kappa \neq 0$. By the assumption, we have $\mathbf{n}_{u_{i}}(\mathbf{u})=-\kappa \mathbf{X}_{u_{i}}(\mathbf{u})$, so that there exists a constant vector $\mathbf{v}$ such that $\mathbf{X}(\mathbf{u})=\mathbf{v}-(1 / \kappa) \mathbf{n}(\mathbf{u})$. Then the vector $\mathbf{v}$ satisfies $\langle\mathbf{v}, \mathbf{v}\rangle=1-1 / \kappa^{2}$ and $\langle\mathbf{X}(\mathbf{u})-\mathbf{v}, \mathbf{X}(\mathbf{u})-\mathbf{v}\rangle=-1 / \kappa^{2}$, so that $\langle\mathbf{X}(\mathbf{u}), \mathbf{v}\rangle=1$ for any $\mathbf{u} \in U$. This means that $M$ is a part of a hyperquadric in de Sitter space $H P(\mathbf{v},+1)$. Therefore we have (1), (2) and (4).

On the other hand, if $\bar{\kappa}+1=\kappa=0$ then there exists a constant timelike vector $\mathbf{v}$ such that $\mathbf{n}(\mathbf{u})=\mathbf{v}$ for any $\mathbf{u} \in U$. So that $\langle\mathbf{X}(\mathbf{u}), \mathbf{v}\rangle=$ $\langle\mathbf{X}(\mathbf{u}), \mathbf{n}(\mathbf{u})\rangle=0$ for any $\mathbf{u} \in U$. This means that $M \subset H P(\mathbf{v}, 0)$ Therefore (3) holds. This completes the proof.

We now consider the following Weingarten type formula. Since $\left\{\mathbf{X}_{u_{i}}\right\}_{i=1}^{n-r}$ spans a spacelike vector subspace, we induce a Riemannian metric (the horospherical first fundamental form) by $d s^{2}=\sum_{i, j=1}^{n-r} g_{i j} d u_{i} d u_{j}$ on $M=\mathbf{X}(U)$, where $g_{i j}=\left\langle\mathbf{X}_{u_{i}}, \mathbf{X}_{u_{j}}\right\rangle$. We respectively define the second fundamental invariant and horospherical second fundamental invariant with respect to the timelike unit normal vector field $\mathbf{n}$ by $h_{i j}(\mathbf{n})=-\left\langle\mathbf{n}_{u_{i}}, \mathbf{X}_{u_{j}}\right\rangle$ and $\bar{h}_{i j}(\mathbf{n})=$ $-\left\langle\mathbf{X}_{u_{i}}+\mathbf{n}_{u_{i}}, \mathbf{X}_{u_{j}}\right\rangle$. We have the relation

$$
\bar{h}_{i j}(\mathbf{n})=-g_{i j}+h_{i j}(\mathbf{n}) \quad(\text { for } i, j=1, \ldots, n-r) .
$$

Under the above notations, we have the following Weingarten type formula with respect to the timelike unit normal vector field $\mathbf{n}$, which is anal- 
ogous to ([6], Proposition 3.2)

$$
\pi^{T} \circ(\mathbf{X}+\mathbf{n})_{u_{i}}=-\sum_{k=1}^{n-r} \bar{h}_{i}^{j}(\mathbf{n}) \mathbf{X}_{u_{j}},
$$

where $\left(\bar{h}_{i}^{j}(\mathbf{n})\right)_{i j}=\left(\bar{h}_{i k}(\mathbf{n})\right)_{i k}\left(g^{k j}\right)_{k j}$ and $\left(g^{k j}\right)=\left(g_{k j}\right)^{-1}$. Therefore, the Gauss-Kronecker curvature with respect to $\mathbf{n}$ is given by

$$
K_{h}(\mathbf{n})=\operatorname{det}\left(\bar{h}_{i k}(\mathbf{n})\right) / \operatorname{det}\left(g_{k j}\right) .
$$

Since $\left\langle\mathbf{X}+\mathbf{n}, \mathbf{X}_{u_{j}}\right\rangle \equiv 0$, the coefficients of the second fundamental invariant with respect to the timelike parallel unit normal vector field $\mathbf{n}$ are expressed by

$$
\begin{aligned}
\bar{h}_{i j}(\mathbf{n}) & =-\left\langle\mathbf{X}_{u_{i}}+\mathbf{n}_{u_{i}}, \mathbf{X}_{u_{j}}\right\rangle \\
& =-\partial\left\langle\mathbf{X}+\mathbf{n}, \mathbf{X}_{u_{j}}\right\rangle / \partial u_{i}+\left\langle\mathbf{X}+\mathbf{n}, \mathbf{X}_{u_{i} u_{j}}\right\rangle \\
& =\left\langle\mathbf{X}+\mathbf{n}, \mathbf{X}_{u_{i} u_{j}}\right\rangle .
\end{aligned}
$$

Therefore the horospherical second fundamental invariant at a point $p_{0}=$ $\mathbf{X}\left(\mathbf{u}_{0}\right)$ depends only on the timelike vector $\mathbf{n}_{0}=\mathbf{n}\left(\mathbf{u}_{0}\right)$. It is independent of the choice of timelike parallel unit normal vector field $\mathbf{n}$ with $\mathbf{n}_{0}=\mathbf{n}\left(\mathbf{u}_{0}\right)$.

Let $\mathbf{n}_{0}$ be a timelike unit normal vector. We say that a point $p_{0}=\mathbf{X}\left(\mathbf{u}_{0}\right)$ is an $\mathbf{n}_{0}$-parabolic point (resp. $\mathbf{n}_{0}$-umbilic point) of $M$ if $K_{h}(\mathbf{n})\left(\mathbf{u}_{0}\right)=0$ $\left(S_{p_{0}}(\mathbf{n})=\bar{\kappa}_{p_{0}}(\mathbf{n}) \operatorname{id}_{T_{p_{0}} M}\right)$ for some timelike parallel unit normal vector field $\mathbf{n}$ with $\mathbf{n}\left(\mathbf{u}_{0}\right)=\mathbf{n}_{0}$. We also say that $p_{0}$ is an $\mathbf{n}_{0}$-horospherical point if it is an $\mathbf{n}_{0}$-parabolic point and an $\mathbf{n}_{0}$-umbilic point.

\section{Horospherical hypersurfaces and horospherical height functions}

In this section we introduce the notions of horospherical height function and horospherical hypersurface.

Let $\mathbf{X}: U \rightarrow S_{1}^{n}$ be a spacelike submanifolds of codimension $r \geq 2$ in de Sitter space and $p=\mathbf{X}(\mathbf{u})$. We choose unit orthonormal sections

$$
N_{p}(M)=\left\langle\mathbf{X}(\mathbf{u}), \mathbf{n}_{0}(\mathbf{u}), \mathbf{n}_{1}(\mathbf{u}), \ldots, \mathbf{n}_{r-1}(\mathbf{u})\right\rangle_{\mathbb{R}},
$$

where $\mathbf{n}_{0}(\mathbf{u})$ is a timelike unit normal vector and $\mathbf{n}_{i}(\mathbf{u})$ for $i=1, \ldots, r-1$ are spacelike unit normal vectors. We define a map e : $U \times H^{r-1}(-1) \rightarrow$ $H^{n-1}(-1)$ by

$$
\mathbf{e}(\mathbf{u}, \bar{\mu})=\mu_{0} \mathbf{n}_{0}(\mathbf{u})+\sum_{i=1}^{r-1} \mu_{i} \mathbf{n}_{i}(\mathbf{u}),
$$

where $\bar{\mu}=\left(\mu_{0}, \ldots, \mu_{r-1}\right)$. Let $\theta$ be a fixed real number, we also define a $\operatorname{map} \overline{\mathbf{X}}_{\theta}: U \times H^{r-1}(-1) \rightarrow S_{1}^{n}$ by

$$
\overline{\mathbf{X}}_{\theta}(\mathbf{u}, \bar{\mu})=\cosh \theta \mathbf{X}(\mathbf{u})+\sinh \theta \mathbf{e}(\mathbf{u}, \bar{\mu}) .
$$


We remark that for any spacelike submanifold $\mathbf{X}$ and point $\left(\mathbf{u}_{0}, \bar{\mu}_{0}\right) \in U \times$ $H^{r-1}(-1)$, there are a real number $\theta \neq 0$ and an open neighborhood $V$ of $\left(\mathbf{u}_{0}, \bar{\mu}_{0}\right)$ such that $\overline{\mathbf{X}}_{\theta}$ is spacelike embedding on $V$. We assume that for any $(\mathbf{u}, \bar{\mu}) \in V$ then $(\mathbf{u},-\bar{\mu}) \in V$. We write $C M$ as an image $\overline{\mathbf{X}}_{\theta}(V)$ and call it a spacelike canal hypersurface of $M=\mathbf{X}(U)$. Izumiya, Pei, Romero Fuster and Takahashi [6] introduced the notion of canal surfaces of submanifolds in the hyperbolic space.

We now consider the horospherical height function on a spacelike submanifold. For a spacelike submanifolds $\mathbf{X}$ of codimension $r$, we define the family of functions

$$
H: U \times L C^{*} \rightarrow \mathbb{R}
$$

by $H(\mathbf{u}, \mathbf{v})=\langle\mathbf{X}(\mathbf{u}), \mathbf{v}\rangle-1$, and we call $H$ a horospherical height function on $M$. For $\mathbf{v}_{0} \in L C^{*}$ we denote $h_{\mathbf{v}_{0}}(\mathbf{u})=\left\langle\mathbf{X}(\mathbf{u}), \mathbf{v}_{0}\right\rangle-1$. We have the following proposition which is analogous to ([6], Proposition 3.4).

Proposition 4.1. Let $H: U \times L C^{*} \rightarrow \mathbb{R}$ be a horospherical height function of a spacelike submanifold $X: U \rightarrow S_{1}^{n}$ of codimension $r$. Then $H(\mathbf{u}, \mathbf{v})=$ $\partial H(\mathbf{u}, \mathbf{v}) / \partial u_{i}=0$ for $i=1, \ldots, n-r$ if and only if $\mathbf{v}=\mathbf{X}(\mathbf{u})+\mathbf{e}(\mathbf{u}, \bar{\mu})$ for some $\bar{\mu} \in H^{r-1}(-1)$.

The proof of the above proposition is similar to that of Proposition 3.4 in [6], so it is omitted. The discriminant set of the horospherical height function $H$ is

$$
D_{H}=\left\{\mathbf{X}(\mathbf{u})+\mathbf{e}(\mathbf{u}, \bar{\mu}) \mid(\mathbf{u}, \bar{\mu}) \in U \times H^{r-1}(-1)\right\} .
$$

We define a map $H S_{\mathbf{X}}: U \times H^{r-1}(-1) \rightarrow L C^{*}$ by

$$
H S_{\mathbf{X}}(\mathbf{u}, \bar{\mu})=\mathbf{X}(\mathbf{u})+\mathbf{e}(\mathbf{u}, \bar{\mu}),
$$

which we call a horospherical hypersurface of $M$. We remark that $H S_{\mathbf{X}}$ depends on the choice of the orthonormal frames of $N(M)$.

Let $\left\{\mathbf{X}, \mathbf{n}_{0}, \ldots, \mathbf{n}_{r-1}\right\}$ and $\left\{\mathbf{X}, \mathbf{n}_{0}^{\prime}, \ldots, \mathbf{n}_{r-1}^{\prime}\right\}$ be two orthonormal frames of $N(M)$ with $\mathbf{n}_{0}, \mathbf{n}_{0}^{\prime} \in H_{+}^{n-1}(-1)$. Then we have $\mathbf{n}_{i}=\sum_{j=0}^{r-1} \lambda_{i}^{j} \mathbf{n}_{j}^{\prime}$, where

$$
\lambda_{i}^{j}(\mathbf{u})= \begin{cases}-\left\langle\mathbf{n}_{i}, \mathbf{n}_{j}^{\prime}\right\rangle & \text { if } j=0, \\ \left\langle\mathbf{n}_{i}, \mathbf{n}_{j}^{\prime}\right\rangle & \text { if } j=1, \ldots, r-1 .\end{cases}
$$

Then we have a diffeomorphism $\Phi: U \times H^{r-1}(-1) \rightarrow U \times H^{r-1}(-1)$ defined by

$$
\Phi(\mathbf{u}, \bar{\mu})=\left(\mathbf{u},\left(\sum_{i=0}^{r-1} \lambda_{i}^{0}(\mathbf{u}) \mu_{i}, \ldots, \sum_{i=0}^{r-1} \lambda_{i}^{r-1}(\mathbf{u}) \mu_{i}\right)\right)
$$


We also define $\mathbf{e}^{\prime}(\mathbf{u}, \bar{\mu})=\sum_{i=0}^{r-1} \mu_{i} \mathbf{n}_{i}^{\prime}(\mathbf{u})$. It follows from the above that $\mathbf{e}(\mathbf{u}, \bar{\mu})=\mathbf{e}^{\prime} \circ \Phi(\mathbf{u}, \bar{\mu})$. Therefore we have

$$
H S_{\mathbf{X}}(\mathbf{u}, \bar{\mu})=H S_{\mathbf{X}}^{\prime} \circ \Phi(\mathbf{u}, \bar{\mu})
$$

where $H S_{\mathbf{X}}^{\prime}=\mathbf{X}(\mathbf{u})+\mathbf{e}^{\prime}(\mathbf{u}, \bar{\mu})$. This means that $H S \mathbf{X}$ is independent to the choice of orthonormal frames of $N(M)$ up to the diffeomorphic parametrization. We have a following proposition which is analogous to ([6], Proposition 3.5).

Proposition 4.2. Let $\mathbf{X}: U \rightarrow S_{1}^{n}$ be a spacelike hypersurface of codimension $r \geq 2$ in de Sitter space, then $H S_{\mathbf{X}}(\mathbf{u}, \bar{\mu})=\mathbf{X}(\mathbf{u})+\mathbf{e}(\mathbf{u}, \bar{\mu})$ is constant map for some smooth map $\bar{\mu}: U \rightarrow H^{r-1}(-1)$ if and only if $M$ is a part of de Sitter hyperhorosphere $H P(\mathbf{v}, 1) \cap S_{1}^{n}$. By Proposition 3.1, if $M$ is totally $\mathbf{e}(\mathbf{u}, \bar{\mu}(\mathbf{u}))$-umbilic for some parallel normal vector field $\mathbf{e}(\mathbf{u}, \bar{\mu}(\mathbf{u}))$ and $K_{h}(\mathbf{e}(\mathbf{u}, \vec{\mu}(\mathbf{u})))(\mathbf{u})=0$, then the above assertion holds.

Proof. Suppose that $\mathbf{v}_{0}=\mathbf{X}(\mathbf{u})+\mathbf{e}(\mathbf{u}, \bar{\mu})$ is a constant vector. Since $\mathbf{e}(\mathbf{u}, \bar{\mu})$ is pseudo orthogonal to $\mathbf{X}(\mathbf{u})$, then we have $\left\langle\mathbf{X}(\mathbf{u}), \mathbf{v}_{\mathbf{0}}\right\rangle=+1$ for any $\mathbf{u} \in U$. This means that $M$ is a part of a de Sitter hyperhorosphere $H P\left(\mathbf{v}_{0}, 1\right) \cap S_{1}^{n}$. On the other hand, if $M \subset H P\left(\mathrm{v}_{0}, 1\right) \cap S_{1}^{n}$ for some lightlike vector, then $\left\langle\mathbf{v}_{0}-\mathbf{X}(\mathbf{u}), \mathbf{X}(\mathbf{u})\right\rangle=0$ for any $\mathbf{u} \in U$. Since $\mathbf{X}(\mathbf{u})$ is pseudo orthogonal to $\mathbf{X}_{u_{i}}(\mathbf{u})$, it follows that $\left\langle\mathbf{v}_{0}-\mathbf{X}(\mathbf{u}), \mathbf{X}_{u_{i}}(\mathbf{u})\right\rangle=0$. This means that $\mathbf{X}(\mathbf{u})-\mathbf{v}_{0}$ is a normal vector of $M$ at $p=\mathbf{X}(\mathbf{u})$. We define a function $\bar{\mu}(\mathbf{u})$ by

$$
\bar{\mu}(\mathbf{u})=-\left\langle\mathbf{X}(\mathbf{u})-\mathbf{v}_{\mathbf{0}}, \mathbf{n}_{0}(\mathbf{u})\right\rangle \mathbf{n}_{0}(\mathbf{u})+\sum_{i=1}^{r-1}\left\langle\mathbf{X}(\mathbf{u})-\mathbf{v}_{\mathbf{0}}, \mathbf{n}_{i}(\mathbf{u})\right\rangle \mathbf{n}_{i}(\mathbf{u}) .
$$

Then we have $\mathbf{v}_{0}-\mathbf{X}(\mathbf{u})=\mathbf{e}(\mathbf{u}, \bar{\mu})$. This completes the proof.

Since the image of $H S_{\mathbf{x}}$ is the discriminant set of the horospherical height function $H$ on $M$, the singular set of $H S_{\mathbf{X}}$ corresponds to the null set of the Hessian matrix of the horospherical height function with the fixed parameter $\mathbf{v}$ at each point. Therefore we have the following proposition which is analogous to ([6], Proposition 3.6).

Proposition 4.3. The singular set of $H S_{\mathbf{X}}$ is given by

$$
\Sigma\left(H S_{\mathbf{X}}\right)=\left\{(\mathbf{u}, \bar{\mu}) \in U \times H^{r-\mathbf{l}}(-1) \mid K_{h}(\mathbf{e}(\mathbf{u}, \bar{\mu}))(\mathbf{u})=0\right\} .
$$

Proof. Let $h_{\mathbf{v}}(\mathbf{u})$ be a horospherical height function with $\mathbf{v} \in L C^{*}$, then we have Hess $h_{\mathbf{v}}(\mathbf{u})=\left\langle\mathbf{X}_{u_{i} u_{j}}(\mathbf{u}), \mathbf{v}\right\rangle$. Suppose that $(\mathbf{u}, \mathbf{v}) \in \Sigma_{*}(H)$, then $\mathbf{v}=\mathbf{X}(\mathbf{u})+\mathbf{e}(\mathbf{u}, \bar{\mu})$ for some $\bar{\mu} \in H^{r-1}(-1)$. We recall that $\bar{h}_{i j}(\mathbf{v})(\mathbf{u})=$ $\left\langle\mathbf{X}_{u_{i} u_{j}}(\mathbf{u}), \mathbf{X}(\mathbf{u})+\mathbf{e}(\mathbf{u}, \bar{\mu})\right\rangle$, where $\left(\bar{h}_{i j}(\mathbf{v})(\mathbf{u})\right)$ is the horospherical second fundamental invariant with respect to the timelike direction $\mathbf{e}(\mathbf{u}, \bar{\mu})$. The horospherical Gauss-Kronecker curvature is 


$$
\begin{aligned}
K_{h}(\mathbf{e}(\mathbf{u}, \bar{\mu}))(\mathbf{u}) & =\operatorname{det}\left(\left\langle\mathbf{X}_{u_{i} u_{j}}(\mathbf{u}), \mathbf{X}(\mathbf{u})+\mathbf{e}(\mathbf{u})\right\rangle\right) / \operatorname{det}\left(g_{i j}(\mathbf{u})\right) \\
& =\operatorname{det} \operatorname{Hess} h_{\mathbf{v}}(\mathbf{u}) / \operatorname{det}\left(g_{i j}(\mathbf{u})\right),
\end{aligned}
$$

where $\left(g_{i j}(\mathbf{u})\right)$ is the first fundamental invariant of $M$. Therefore Hess $h_{\mathbf{v}}(\mathbf{u})$ $=0$ if and only if $K_{h}(\mathbf{e}(\mathbf{u}, \bar{\mu}))(\mathbf{u})=0$. This completes the proof.

The singular set of $H S \mathbf{X}$ corresponds to the parabolic set of $M$ with respect to some timelike parallel normal vector field $\mathbf{e}(\mathbf{u}, \bar{\mu})$. By the proof of above proposition, we have rank Hess $h_{\mathbf{v}_{0}}\left(\mathbf{u}_{0}\right)=\operatorname{rank}\left(\bar{h}_{i j}\left(\mathbf{v}_{0}\right)\left(\mathbf{u}_{0}\right)\right)_{i j}$. Therefore we also have the following proposition which is analogous to ([6], Proposition 3.7).

Proposition 4.4. For any spacelike submanifold $\mathrm{X}$ of codimension $r \geq 2$ and lightlike vector $\mathbf{v}_{0}=\mathbf{X}\left(\mathbf{u}_{0}\right)+\mathbf{e}\left(\mathbf{u}_{0}, \bar{\mu}_{0}\right)$, we have the following assertions.

(1) $A$ point $p_{0}=\mathbf{X}\left(\mathbf{u}_{0}\right)$ is an $\mathbf{e}\left(\mathbf{u}_{0}, \bar{\mu}_{0}\right)$-parabolic point if and only if

$$
\operatorname{det} \operatorname{Hess} h_{\mathbf{v}_{0}}\left(\mathbf{u}_{0}\right)=0 .
$$

(2) A point $p_{0}$ is an $\mathbf{e}\left(\mathbf{u}_{0}, \vec{\mu}_{0}\right)$-horospherical point if and only if

$$
\text { rank Hess } h_{\mathbf{v}_{0}}\left(\mathbf{u}_{0}\right)=0 \text {. }
$$

Here Hess $h_{\mathbf{v}_{0}}\left(\mathbf{u}_{0}\right)$ is a Hessian matrix of $h_{\mathbf{v}_{0}}(\mathbf{u})$ at $\mathbf{u}=\mathbf{u}_{0}$.

We now consider the lightcone height function and the lightcone Gauss image of spacelike canal hypersurface $\overline{\mathbf{X}}_{\theta}: V \rightarrow S_{1}^{n}$ with $V \subset U \times H^{r-1}(-1)$. The lightcone height function $\bar{H}: V \times L C^{*} \rightarrow \mathbb{R}$ of the spacelike hypersurface $\overline{\mathbf{X}}_{\theta}$ is

$$
\vec{H}((\mathbf{u}, \bar{\mu}), \mathbf{v})=\left\langle\overline{\mathbf{X}}_{\theta}(\mathbf{u}, \bar{\mu}), \mathbf{v}\right\rangle-1 .
$$

We denote $\bar{h}_{\mathbf{v}}(\mathbf{u})=\bar{H}((\mathbf{u}, \bar{\mu}), \mathbf{v})$ for any $\mathbf{v} \in L C^{*}$. Now we define a map $\overline{\mathbf{e}}: V \rightarrow H^{n-1}(-1)$ by $\overline{\mathbf{e}}(\mathbf{u}, \bar{\mu})=\sinh \theta \mathbf{X}(\mathbf{u})+\cosh \theta \mathbf{e}(\mathbf{u}, \bar{\mu})$. Then we have $\left\langle\overline{\mathbf{e}}(\mathbf{u}, \bar{\mu}), \overline{\mathbf{X}}_{\theta}(\mathbf{u})\right\rangle=\left\langle\overline{\mathbf{e}}(\mathbf{u}, \bar{\mu}), \overline{\mathbf{X}}_{\theta, u_{i}}(\mathbf{u})\right\rangle=0$ for any $(\mathbf{u}, \bar{\mu}) \in V$ and $i=1, \ldots, n-r$. Therefore $\overline{\mathbf{e}}$ is a timelike normal of $C M$. The positive lightcone Gauss image $\mathbb{L}_{C M}: V \rightarrow L C^{*}$ is defined by

$$
\mathbb{L}_{C M}(\mathbf{u}, \bar{\mu})=\overline{\mathbf{X}}_{\theta}(\mathbf{u})+\overline{\mathbf{e}}(\mathbf{u}, \bar{\mu})=(\cosh \theta+\sinh \theta)(\mathbf{X}(\mathbf{u})+\mathbf{e}(\mathbf{u}, \bar{\mu})) .
$$

By Proposition 2.2, $\bar{H}((\mathbf{u}, \bar{\mu}), \mathbf{v})=\bar{H}_{u_{i}}((\mathbf{u}, \bar{\mu}), \mathbf{v})=\bar{H}_{\mu_{j}}((\mathbf{u}, \bar{\mu}), \mathbf{v})=0$ for $i=1, \ldots, n-r$ and $j=0, \ldots, r-1$ if and only if $\mathbf{v}=\overline{\mathbf{X}}_{\theta}(\mathbf{u}) \pm \overline{\mathbf{e}}(\mathbf{u}, \bar{\mu})=$ $e^{ \pm \theta}(\mathbf{X}(\mathbf{u})+\mathbf{e}(\mathbf{u}, \pm \bar{\mu}))$. By assumption, $(\mathbf{u},-\bar{\mu})$ is also an element of $V$. Therefore the discriminant set of the lightcone height function $\vec{H}$ is

$$
D(\bar{H})=\left\{e^{ \pm \theta}(\mathbf{X}(\mathbf{u})+\mathbf{e}(\mathbf{u}, \bar{\mu})) \mid(\mathbf{u}, \bar{\mu}) \in V\right\} .
$$

We now define a diffeomorphism

$$
\mathcal{M}_{c}: L C^{*} \rightarrow L C^{*}
$$


given by $M_{c}(\mathbf{v})=c \mathbf{v}$ for a fixed positive real number $c$. Then we have the following lemma, which is analogous to ([6], Proposition 3.9).

LEMMA 4.5. Under the above notations, we have

$$
\mathcal{M}_{c} \circ H S_{\mathbf{X}}(\mathbf{u}, \bar{\mu})=\mathbb{L}_{C M}(\mathbf{u}, \bar{\mu})
$$

on $V \subset U \times H^{r-1}(-1)$, where $c=e^{ \pm \theta}$.

By the above lemma, the horospherical hypersurface $H S_{\mathbf{X}}$ is locally diffeomorphic to the lightcone Gauss image of the spacelike canal hypersurface $\overline{\mathbf{X}}_{\theta}$.

\section{Horospherical hypersurfaces as wave fronts}

In this section we naturally interpret the horospherical hypersurfaces of $M$ as a wave front set of the horospherical height functions in the theory of Legendrian singularities.

By proceeding arguments in $\S 2$, the horospherical hypersurface $H S_{\mathbf{X}}$ is the discriminant set of the horospherical height function $H$, and the singular point set of the horospherical hypersurface is the horospherical point set. We have the following proposition which is analogous to ([6], Proposition 4.1).

Proposition 5.1. Let $\mathbf{X}: U \rightarrow S_{1}^{n}$ be a spacelike submanifold of codimension $r \geq 2$ and $H: U \times L C^{*} \rightarrow \mathbb{R}$ be a horospherical height function of $M$. Then $H$ is a Morse family.

Proof. We denote

$$
\begin{aligned}
\mathbf{X}(\mathbf{u}) & =\left(X_{0}(\mathbf{u}), \ldots, X_{n}(\mathbf{u})\right) \quad \text { and } \\
\mathbf{X}_{u_{i}}(\mathbf{u}) & =\left(X_{0, u_{i}}(\mathbf{u}), \ldots, X_{n, u_{i}}(\mathbf{u})\right) .
\end{aligned}
$$

For any $\mathbf{v}=\left(v_{0}, \ldots, v_{n}\right) \in L C^{*}$, we have $v_{0} \neq 0$. Without loss of generality, we assume that $v_{0}=\sqrt{v_{1}^{2}+\cdots+v_{n}^{2}}>0$, so that we have

$$
H(\mathbf{u}, \mathbf{v})=\langle\mathbf{X}(\mathbf{u}), \mathbf{v}\rangle-1=-X_{0} v_{0}+\sum_{i=1}^{n} X_{n} v_{n}-1
$$

We now prove a map

$$
\Delta^{*} H=\left(H, \frac{\partial H}{\partial u_{1}}, \ldots, \frac{\partial H}{\partial u_{n-r}}\right)
$$

is non singular at any $(\mathbf{u}, \mathbf{v}) \in \Sigma_{*}(H)$. The Jacobian matrix of $\Delta^{*} H$ is

$$
J \Delta^{*} H(\mathbf{u}, \mathbf{v})=\left(\begin{array}{c|c}
* & \frac{\partial H}{\partial v_{j}}(\mathbf{u}, \mathbf{v})_{j=1, \ldots, n} \\
\hline * & \left(\frac{\partial^{2} H}{\partial u_{i} \partial v_{j}}(\mathbf{u}, \mathbf{v})\right)_{\substack{j=1, \ldots, n \\
i=1, \ldots, n-r}}
\end{array}\right) .
$$


We denote an $(n-r+1) \times n$ matrix B by $J \Delta^{*} H=(* \mid B)$. It is sufficient to show that $\operatorname{rank} B=n-r+1$ at $(\mathbf{u}, \mathbf{v}) \in \Sigma_{*}(H)$. We also denote an $(n-r+3) \times(n+1)$ matrix $\mathrm{C}$ by

$$
C=\left(\begin{array}{cccc}
1 & 0 & \cdots & 0 \\
v_{0} & v_{1} & \cdots & v_{n} \\
X_{0} & X_{1} & \cdots & X_{n} \\
X_{0, u_{1}} & X_{1, u_{1}} & \cdots & X_{n, u_{1}} \\
\vdots & \vdots & \ddots & \vdots \\
X_{0, u_{n-r}} & X_{1, u_{n-r}} & \cdots & X_{n, u_{n-r}}
\end{array}\right) .
$$

We now show that the rank of the matrix $C$ is equal to $n-r+3$. Since $\mathbf{v}, \mathbf{X}(\mathbf{u})$ and $\mathbf{X}_{u_{i}}(\mathbf{u})$ are linearly independent for all $(\mathbf{u}, \mathbf{v}) \in \Sigma_{*}(H)$, it is sufficient to show that timelike unit vector $e=(1,0, \ldots, 0)$ can not be written by a linear combination of $\mathbf{v}, \mathbf{X}(\mathbf{u})$ and $\mathbf{X}_{u_{i}}(\mathbf{u})$. If that is not so, there exists some real numbers $\eta, \mu, \xi_{i}$ such that $e=\eta \mathbf{v}+\mu \mathbf{X}(\mathbf{u})+w$ and $w=\sum_{i=1}^{n-r} \xi_{i} \mathbf{X}_{u_{i}}(\mathbf{u})$. Then we have $\langle e, e\rangle=\mu^{2}+\langle w, w\rangle$. However, $w$ is a spacelike vector, so that $\langle e, e\rangle$ would not be negative, which contradicts our assumption. This means that $e, \mathbf{v}, \mathbf{X}(\mathbf{u})$ and $\mathbf{X}_{u_{i}}(\mathbf{u})$ are linearly independent, therefore we have $\operatorname{rank} C=n-r+3$.

We now show $\operatorname{rank} B=\operatorname{rank} C^{\prime}-2$. We subtract the second row multiplied by $X_{0} / v_{0}$ from the third row of the matrix $C$, and add the second row multiplied by $X_{0, u_{k}}(\mathbf{u}) / v_{0}$ from the $(3+k)$-th row for $k=1, \ldots, n-r$. Then we have a matrix

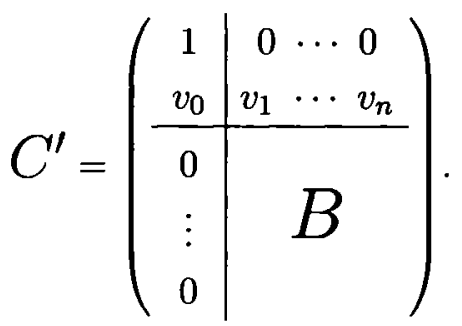

Therefore we have $\operatorname{rank} B=\operatorname{rank} C^{\prime}-2=n-r+1$. This completes the proof.

Since $H$ is a Morse family of hypersurfaces, we have the Legendrian immersion germ $\mathcal{L}_{H}:\left(\Sigma_{*}(H),\left(\mathbf{u}_{0}, \mathbf{v}_{0}\right)\right) \rightarrow P T^{*}\left(L C^{*}\right)$ defined by

$$
\mathcal{L}_{H}(\mathbf{u}, \mathbf{v})=\left(\mathbf{v},\left[\frac{\partial H}{\partial v_{1}}(\mathbf{u}, \mathbf{v}): \ldots: \frac{\partial H}{\partial v_{n}}(\mathbf{u}, \mathbf{v})\right]\right),
$$

where $\left(v_{1}, \ldots, v_{n}\right)$ is the coordinate system of $L C^{*}$.

We remark that the wave front set of the Legendrian immersion germ $\mathcal{L}_{H}$ is the horospherical hypersurfaces $H S_{\mathbf{X}}$ of $M$. On the other hand, we 
define a contact diffeomorphism $\tilde{\mathcal{M}}_{c}: P T^{*}\left(L C^{*}\right) \rightarrow P T^{*}\left(L C^{*}\right)$ by

$$
\tilde{\mathcal{M}}_{c}(\mathbf{v},[\xi])=(c \mathbf{v},[\xi])
$$

where $c$ is a fixed real parameter with $c \neq 0$. By definition, we have the following theorem.

TheOREM 5.2. For a spacelike submanifold $\mathbf{X}: U \rightarrow S_{1}^{n}$, we have

$$
\tilde{\mathcal{M}}_{c} \circ \mathcal{L}_{H}=\mathcal{L}_{\bar{H}}
$$

where $c=e^{ \pm \theta}$ and $\mathcal{L}_{\vec{H}}$ is a Legendrian lift of the lightcone Gauss image $\mathbb{L}_{C M}$ of the spacelike canal hypersurface of $M$.

By the above theorem, the Legendrian lift of the lightcone Gauss image $\mathbb{L}_{C M}$ is $\mathcal{A}$-equivalent to the Legendrian lift of the horospherical hypersurface $H S_{\mathbf{X}}$ of $M$.

\section{Contact with de Sitter hyperhorospheres}

In this section we use the theory of contacts between the spacelike submanifolds and the de Sitter hyperhorospheres, following Montaldi [10].

Let $X_{i}$ and $Y_{i}(i=1,2)$ be submanifolds of $\mathbb{R}^{n}$ with $\operatorname{dim} X_{1}=\operatorname{dim} X_{2}$, $\operatorname{dim} Y_{1}=\operatorname{dim} Y_{2}$ and $y_{i} \in X_{i} \cap Y_{i}$ for $i=1,2$. We say that the contact of $X_{1}$ and $Y_{1}$ at $y_{1}$ is the same type as the contact of $X_{2}$ and $Y_{2}$ at $y_{2}$ if there is a diffeomorphism germ $\Phi:\left(\mathbb{R}^{n}, y_{1}\right) \rightarrow\left(\mathbb{R}^{n}, y_{2}\right)$ such that $\Phi\left(\left(X_{1}, y_{1}\right)\right)=$ $\left(X_{2}, y_{2}\right)$ and $\Phi\left(\left(Y_{1}, y_{1}\right)\right)=\left(Y_{2}, y_{2}\right)$. In this case we write $K\left(X_{1}, Y_{1} ; y_{1}\right)=$ $K\left(X_{2}, Y_{2} ; y_{2}\right)$. Two function germs $g_{1}, g_{2}:\left(\mathbb{R}^{n}, a_{i}\right) \rightarrow(\mathbb{R}, 0)(i=1,2)$ are $\mathcal{K}$-equivalent if there are a diffeomorphism germ $\Phi:\left(\mathbb{R}^{n}, a_{1}\right) \rightarrow\left(\mathbb{R}^{n}, a_{2}\right)$ and a function germ $\lambda:\left(\mathbb{R}^{n}, a_{1}\right) \rightarrow \mathbb{R}$ with $\lambda\left(a_{1}\right) \neq 0$ such that $f_{1}=\lambda \cdot\left(g_{2} \circ \Phi\right)$. In [10] Montaldi has shown the following theorem.

Theorem 6.1. ([10]) Let $X_{i}$ and $Y_{i}(i=1,2)$ be submanifolds of $\mathbb{R}^{n}$ with $\operatorname{dim} X_{1}=\operatorname{dim} X_{2}, \operatorname{dim} Y_{1}=\operatorname{dim} Y_{2}$ and $y_{i}=X_{i} \cap Y_{i}$ for $i=1,2$. Let $g_{i}:\left(X_{i}, x_{i}\right) \rightarrow\left(\mathbb{R}^{n}, y_{i}\right)$ be immersion germs and $f_{i}:\left(\mathbb{R}^{n}, y_{i}\right) \rightarrow(\mathbb{R}, 0)$ be submersion germs with $\left(Y_{i}, y_{i}\right)=\left(f_{i}^{-1}(0), y_{i}\right)$. Then $K\left(X_{1}, Y_{1} ; y_{1}\right)=$ $K\left(X_{2}, Y_{2} ; y_{2}\right)$ if and only if $f_{1} \circ g_{1}$ and $f_{2} \circ g_{2}$ are $\mathcal{K}$-equivalent.

We now consider the function $\mathcal{H}: S_{1}^{n} \times L C^{*} \rightarrow \mathbb{R}$ defined by $\mathcal{H}(x, \mathbf{v})=$ $\langle x, \mathbf{v}\rangle-1$. Given $\mathbf{v}_{0} \in L C^{*}$, we denote $\mathfrak{h}_{\mathbf{v}_{0}}(x)=\mathcal{H}\left(x, \mathbf{v}_{0}\right)$, so that we have $\mathfrak{h}_{\mathbf{v}_{0}}^{-1}(0)=H P\left(\mathbf{v}_{0},+1\right) \cap S_{1}^{n}$. Let $\mathbf{X}: U \rightarrow \mathbb{R}_{1}^{n}$ be a spacelike submanifold of codimension $r \geq 2$. For any $\mathbf{u}_{0} \in U$ and $\bar{\mu}_{0} \in H^{r-1}(-1)$, we take a point $\mathbf{v}_{0}=\mathbf{X}\left(\mathbf{u}_{0}\right)+\mathbf{e}\left(\mathbf{u}_{0}, \bar{\mu}_{0}\right)$. By Proposition 4.1, we have

$$
\begin{array}{r}
\left(\mathfrak{h}_{\mathbf{v}_{0}} \circ \mathbf{X}\right)\left(\mathbf{u}_{0}\right)=\mathcal{H} \circ\left(\mathbf{X} \times \operatorname{id}_{L C^{*}}\right)\left(\mathbf{u}_{0}, \mathbf{v}_{0}\right)=H\left(\mathbf{u}_{0}, \mathbf{v}_{0}\right)=0 \\
\frac{\partial\left(\mathfrak{h}_{\mathbf{v}_{0}} \circ \mathbf{X}\right)}{\partial u_{i}}\left(\mathbf{u}_{0}\right)=\frac{\partial H}{\partial u_{i}}\left(\mathbf{u}_{0}, \mathbf{X}\left(\mathbf{u}_{0}\right)+\mathbf{e}\left(\mathbf{u}_{0}, \bar{\mu}_{0}\right)\right)=0 .
\end{array}
$$


It follows that the de Sitter hyperhorosphere $\mathfrak{h}_{\mathbf{v}_{0}}^{-1}(0)=H P\left(\mathbf{v}_{0},+1\right) \cap S_{1}^{n}$ is tangent to $M$ at $p_{0}=\mathbf{X}\left(\mathbf{u}_{0}\right)$. In this case we call $H P\left(\mathbf{v}_{0},+1\right) \cap S_{1}^{n}$ a tangent de Sitter hyperhorosphere (briefly, tangent hyperhorosphere) with respect to $\mathbf{X}\left(\mathbf{u}_{0}\right)+\mathbf{e}\left(\mathbf{u}_{0}, \bar{\mu}_{0}\right)$. We may also consider the contacts of the spacelike canal surface $C M=\overline{\mathbf{X}}(V)$ and the de Sitter hyperhorospheres. (see [7])

We now review some notions of Legendrian singularity theory to study the contact between hypersurfaces and de Sitter hyperhorospheres. We say that Legendrian immersion germs $\iota_{i}:\left(U_{i}, \mathbf{u}_{i}\right) \rightarrow\left(P T^{*} \mathbb{R}^{n}, p_{i}\right)(i=1,2)$ are Legendrian equivalent if there are a contact diffeomorphism germ $H$ : $\left(P T^{*} \mathbb{R}^{n}, p_{1}\right) \rightarrow\left(P T^{*} \mathbb{R}^{n}, p_{2}\right)$ and a diffeomorphic germ $\tau:\left(U_{1}, \mathbf{u}_{1}\right) \rightarrow$ $\left(U_{2}, \mathbf{u}_{2}\right)$ such that $H$ preserves fibers of $\pi$ and $H \circ \iota_{1}=\iota_{2} \circ \tau$. A Legendrian immersion germ at a point is said to be Legendrian stable if for every map with the given germ there are a neighborhood in the space of Legendrian immersions (in the Whitney $C^{\infty}$-topology) and a neighborhood of the original point such that each Legendrian map belonging to the first neighborhood has in the second neighborhood a point at which its germ is Legendrian equivalent to the original germ. (see [1]).

Proposition 6.2. ([13]) Let $i_{1}, i_{2}$ be Legendrian immersion germs such that regular sets of $\pi \circ i_{1}$ and $\pi \circ i_{2}$ are respectively dense. Then $i_{1}, i_{2}$ are Legendrian equivalent if and only if corresponding wave front sets $W\left(i_{1}\right)$ and $W\left(i_{2}\right)$ are diffeomorphic as set germs.

Let $F_{i}:\left(\mathbb{R}^{n} \times \mathbb{R}^{k},\left(a_{i}, b_{i}\right)\right) \rightarrow(\mathbb{R}, c)(k=1,2)$ be $k$-parameter unfoldings of function germs $f_{i}$. We say that $F_{1}$ and $F_{2}$ are $\mathcal{P}$ - $\mathcal{K}$-equivalent if there exists a diffeomorphism germ $\Phi:\left(\mathbb{R}^{n} \times \mathbb{R}^{k},\left(a_{1}, b_{1}\right)\right) \rightarrow\left(\mathbb{R}^{n} \times \mathbb{R}^{k},\left(a_{2}, b_{2}\right)\right)$ of the form $\Phi(\mathbf{u}, x)=\left(\phi_{1}(\mathbf{u}, x), \phi_{2}(x)\right)$ for $(\mathbf{u}, x) \in \mathbb{R}^{n} \times \mathbb{R}^{k}$ and a function germ $\lambda:\left(\mathbb{R}^{n} \times \mathbb{R}^{k},\left(a_{1}, b_{1}\right)\right) \rightarrow \mathbb{R}$ such that $\lambda\left(a_{1}, b_{1}\right) \neq 0$ and $F_{1}(\mathbf{u}, x)=$ $\lambda(\mathbf{u}, x) \cdot\left(F_{2} \circ \Phi\right)(\mathbf{u}, x)$.

Theorem 6.3. ([1, 12]) Let $F, G:\left(\mathbb{R}^{k} \times \mathbb{R}^{n}, \mathbf{0}\right) \rightarrow(\mathbb{R}, \mathbf{0})$ be Morse families and denote the corresponding Legendrian immersion germs by $\mathcal{L}_{F}, \mathcal{L}_{G}$. Then

(1) $\mathcal{L}_{F}$ and $\mathcal{L}_{G}$ are Legendrian equivalent if and only if $F$ and $G$ are $\mathcal{P}_{-} \mathcal{K}$ equivalent.

(2) $\mathcal{L}_{F}$ is Legendrian stable if and only if $F$ is $\mathcal{K}$-versal deformation of $f$.

Let $G_{i}:\left(\mathbb{R}^{m}, a_{i}\right) \rightarrow\left(\mathbb{R}^{n}, b_{i}\right)$ (for $\left.i=1,2\right)$ be map germs. We say that $G_{1}$ and $G_{2}$ are $\mathcal{A}$-equivalent if and only if there exist diffeomorphism germs $\phi:\left(\mathbb{R}^{m}, a_{1}\right) \rightarrow\left(\mathbb{R}^{m}, a_{2}\right)$ and $\Phi:\left(\mathbb{R}^{n}, b_{1}\right) \rightarrow\left(\mathbb{R}^{n}, b_{2}\right)$ such that $\Phi \circ G_{1}=G_{2} \circ \phi$.

We denote $h_{i, \mathbf{v}_{i}}:\left(U, \mathbf{u}_{i}\right) \rightarrow(\mathbb{R}, \mathbf{0})(i=1,2)$ by $h_{i, \mathbf{v}_{i}}(\mathbf{u})=H_{i}\left(\mathbf{u}, \mathbf{v}_{i}\right)$. Then we have $h_{i, \mathbf{v}_{i}}(\mathbf{u})=\left(\mathfrak{h}_{i, \mathbf{v}_{i}} \circ \mathbf{X}_{i}\right)(\mathbf{u})$. By Theorem 6.1, 


$$
K\left(\mathbf{X}_{\mathbf{1}}(U), H P\left(\mathbf{v}_{1}, 1\right) \cap S_{1}^{n} ; p_{1}\right)=K\left(\mathbf{X}_{\mathbf{2}}(U), H P\left(\mathbf{v}_{2}, 1\right) \cap S_{1}^{n} ; p_{2}\right)
$$

if and only if $h_{1, \mathbf{v}_{1}}$ and $h_{2, \mathbf{v}_{2}}$ are $\mathcal{K}$-equivalent.

Let $Q\left(\mathbf{X}, \mathbf{u}_{0}\right)$ be the local ring of the horospherical height function germ $h_{\mathbf{v}_{0}}:\left(U, \mathbf{u}_{0}\right) \rightarrow \mathbb{R}$ defined by

$$
Q\left(\mathbf{X}, \mathbf{u}_{0} ; \bar{\mu}_{0}\right)=C_{\mathbf{u}_{0}}^{\infty}(U) /\left\langle h_{\mathbf{v}_{0}}\right\rangle_{C_{\mathbf{u}_{0}}^{\infty}(U)},
$$

where $\mathbf{v}_{0}=\mathbf{X}\left(\mathbf{u}_{0}\right)+\mathbf{e}\left(\mathbf{u}_{0}, \bar{\mu}_{0}\right), \bar{\mu}_{0} \in H^{r-1}(-1)$ and $C_{\mathbf{u}_{0}}^{\infty}(U)$ is the local ring of function germs at $\mathbf{u}_{0}$ with the unique maximal ideal $\mathfrak{M}$. We also denote $Q\left(\overline{\mathbf{X}}_{\theta},\left(\mathbf{u}_{0}, \bar{\mu}_{0}\right)\right)$ as the local ring of the lightcone height function germ $\bar{h}_{\mathbf{v}_{0}^{\prime}}:\left(U \times H^{r-1}(-1),\left(\mathbf{u}_{0}, \bar{\mu}_{0}\right)\right) \rightarrow(\mathbb{R}, 0)$ of the canal hypersurface $\overline{\mathbf{X}}_{\theta}$, where $\mathbf{v}_{0}^{\prime}=\mathbb{L}_{C M}\left(\mathbf{u}_{0}, \bar{\mu}_{0}\right)$.

Proposition 6.4. ([3], Proposition A.4) Let $F, G:\left(\mathbb{R}^{k} \times \mathbb{R}^{n}, \mathbf{0}\right) \rightarrow(\mathbb{R}, \mathbf{0})$ be Morse families. Suppose that Legendrian immersion germs $\mathcal{L}_{F}$ and $\mathcal{L}_{G}$ are Legendrian stable, then the following conditions are equivalent:

(1) $\left(W\left(\mathcal{L}_{F}\right), \lambda\right)$ and $\left(W\left(\mathcal{L}_{G}\right), \lambda^{\prime}\right)$ are diffeomorphic as set germs.

(2) $\mathcal{L}_{F}$ and $\mathcal{L}_{G}$ are Legendrian equivalent.

(3) $Q(f)$ and $Q(g)$ are isomorphic as $\mathbb{R}$-algebras, where $f=\left.F\right|_{\mathbb{R}^{k} \times\{0\}}$ and $g=\left.G\right|_{\mathbb{R}^{k} \times\{\mathbf{0}\}}$.

We have following theorem.

Theorem 6.5. Let $\mathbf{X}_{i}:\left(U_{i}, \mathbf{u}_{i}\right) \rightarrow\left(S_{1}^{n}, p_{i}\right)(i=1,2)$ be spacelike submanifold germs of codimension at least two in de Sitter space. For $\bar{\mu}_{i} \in$ $H^{r-1}(-1)(i=1,2)$, we denote $\mathbf{v}_{i}=H S_{i}\left(\mathbf{u}_{i}, \bar{\mu}_{i}\right), \mathbf{v}_{i}^{\prime}=\mathbb{L}_{C M_{i}}\left(\mathbf{u}_{i}, \bar{\mu}_{i}\right)$, $h_{i, \mathbf{v}_{i}}=\left.H_{i}\right|_{U \times\left\{\mathbf{v}_{i}\right\}}, \bar{h}_{i, \mathbf{v}_{i}^{\prime}}=\left.\bar{H}_{i}\right|_{U \times\left\{\mathbf{v}_{i}^{\prime}\right\}}$ and $p_{i}^{\prime}=\overline{\mathbf{X}}_{i, \theta_{i}}\left(\mathbf{u}_{i}, \bar{\mu}_{i}\right)$. If the corresponding Legendrian immersion germs $\mathcal{L}_{H_{i}}$ are Legendrian stable, then the following conditions are equivalent.

(1) Horospherical hypersurface germs $H S_{\mathbf{X}_{1}}$ and $H S_{\mathbf{X}_{2}}$ are $\mathcal{A}$-equivalent.

(2) Legendrian immersion germs $\mathcal{L}_{H_{1}}$ and $\mathcal{L}_{\mathrm{H}_{2}}$ are Legendrian equivalent.

(3) Horospherical height function germs $H_{1}$ and $H_{2}$ are $\mathcal{P}-\mathcal{K}$-equivalent.

(4) $h_{1, \mathbf{v}_{1}}$ and $h_{2, \mathbf{v}_{2}}$ are $\mathcal{K}$-equivalent.

(5) $K\left(\mathbf{X}_{\mathbf{1}}(U), H P\left(\mathbf{v}_{1}, 1\right) \cap S_{1}^{n} ; p_{1}\right)=K\left(\mathbf{X}_{\mathbf{2}}(U), H P\left(\mathbf{v}_{2}, 1\right) \cap S_{1}^{n} ; p_{2}\right)$.

(6) $Q\left(\mathbf{X}_{1}, \mathbf{u}_{\mathbf{1}}\right)$ and $Q\left(\mathbf{X}_{2}, \mathbf{u}_{\mathbf{2}}\right)$ are isomorphic as $\mathbb{R}$-algebras.

(7) Lightcone Gauss image germs $\mathbb{L}_{C M_{1}}$ and $\mathbb{L}_{C M_{2}}$ are $\mathcal{A}$-equivalent.

(8) Legendrian immersion germs $\mathcal{L}_{\bar{H}_{1}}$ and $\mathcal{L}_{\bar{H}_{2}}$ are Legendrian equivalent.

(9) Lightcone height function germs $\bar{H}_{1}$ and $\vec{H}_{2}$ are $\mathcal{P}-\mathcal{K}$-equivalent.

(10) $\vec{h}_{1, \mathbf{v}_{1}^{\prime}}$ and $\bar{h}_{2, \mathbf{v}_{2}^{\prime}}$ are $\mathcal{K}$-equivalent.

(11) $K\left(C M_{1}, H P\left(\mathbf{v}_{1}^{\prime},+1\right) \cap S_{1}^{n} ; p_{1}^{\prime}\right)=K\left(C M_{2}, H P\left(\mathbf{v}_{2}^{\prime},+1\right) \cap S_{1}^{n} ; p_{2}^{\prime}\right)$.

(12) $Q\left(\overline{\mathbf{X}}_{\theta_{1}},\left(\mathbf{u}_{1}, \bar{\mu}_{1}\right)\right)$ and $Q\left(\overline{\mathbf{X}}_{\theta_{2}},\left(\mathbf{u}_{\mathbf{2}}, \bar{\mu}_{2}\right)\right)$ are isomorphic as $\mathbb{R}$-algebras. 
In this case $\left(\mathbf{X}_{1}{ }^{-1}\left(H P\left(\mathbf{v}_{1}, 1\right) \cap S_{1}^{n}\right), \mathbf{u}_{1}\right)$ and $\left(\mathbf{X}_{2}{ }^{-1}\left(H P\left(\mathbf{v}_{2}, 1\right) \cap S_{1}^{n}\right), \mathbf{u}_{2}\right)$ are diffeomorphic as set germs.

Proof. Since $\mathcal{L}_{H_{1}}$ and $\mathcal{L}_{H_{2}}$ are Legendrian stable, regular sets of $H S_{\mathbf{X}_{1}}$ and $H S_{\mathbf{X}_{2}}$ are respectively dense, by applying Proposition 6.2 , the conditions (1) and (2) are equivalent. By Theorem 6.3, the conditions (2) and (3) are equivalent. By the arguments in Theorem 6.1, the conditions (4) and (5) are equivalent. If we assume the condition (3), then the $\mathcal{P}-\mathcal{K}$-equivalence of $H_{i}(i=1,2)$ preserves the $\mathcal{K}$-equivalence of $h_{i, \mathbf{v}_{i}}$, so that the condition (4) holds. Since the local rings $Q\left(\mathbf{X}_{i}, \mathbf{u}_{i}\right)$ are $\mathcal{K}$-invariant, this means that the condition (6) holds. By Proposition 6.4, the condition (6) implies the condition (2). Therefore the statements from (1) to (6) are equivalent.

By Theorem 5.2, (2) and (8) are equivalent. Since $\mathcal{L}_{H_{i}}$ are Legendrian stable, $\mathcal{L}_{\bar{H}_{i}}$ are also Legendrian stable. So that we may similarly show the equivalence of the conditions from (7) to (12). On the other hand, $h_{i, \mathbf{v}_{i}}^{-1}(0)=$ $\left(\mathbf{X}_{i}{ }^{-1}\left(H P\left(\mathbf{v}_{i}, 1\right) \cap S_{1}^{n}\right), \mathbf{u}_{i}\right)$ and $\mathcal{K}$-equivalence preserves the zero level sets, so that $\left(\mathbf{X}_{i}^{-1}\left(H P\left(\mathbf{v}_{i}, 1\right) \cap S_{1}^{n}\right), \mathbf{u}_{i}\right)(i=1,2)$ are diffeomorphic as set germs. This completes the proof.

We consider generic properties of spacelike submanifolds of codimension $r \geq 2$ in $S_{1}^{n}$. Let $U$ be an open subset of $\mathbb{R}^{n-r}$. We consider the space of spacelike embeddings $\operatorname{Sp}-\operatorname{Emb}\left(U, S_{1}^{n}\right)$ with Whitney $C^{\infty}$-topology. We define a function $\mathcal{H}: S_{1}^{n} \times L C^{*} \rightarrow \mathbb{R}$ by $\mathcal{H}(x, \mathbf{v})=\langle x, \mathbf{v}\rangle$, and denote $\mathfrak{h}_{\mathbf{v}}(x)=\mathcal{H}(x, \mathbf{v})$. Then $\mathfrak{h}_{\mathbf{v}}$ is a submersion for any $\mathbf{v} \in L C^{*}$. For spacelike submanifolds $\mathbf{X} \in \operatorname{Sp}-\operatorname{Emb}\left(U, S_{1}^{n}\right)$, we have $H=\mathcal{H} \circ\left(\mathbf{X} \times \operatorname{id}_{L C^{*}}\right)$. We also have the $\ell$-jet extension $j_{1}^{\ell} H: U \times S_{1}^{n} \rightarrow J^{\ell}(U, \mathbb{R})$ defined by $j_{1}^{\ell} H(x, \mathbf{v})=$ $j^{\ell} \mathfrak{h}_{\mathbf{v}}(\mathbf{u})$. We consider the trivialization $J^{\ell}(U, \mathbb{R}) \equiv U \times \mathbb{R} \times J^{\ell}(n-r, 1)$. For any submanifold $Q \subset J^{\ell}(n-r, 1)$, we denote $\widetilde{Q}=U \times\{0\} \times Q$. Then we have the following proposition as a corollary of Lemma 6 of Wassermann [11].

Proposition 6.6. Let $Q$ be a submanifold of $J^{\ell}(n-1,1)$. Then the set

$$
T_{Q}=\left\{x \in S p-E m b\left(U, S_{1}^{n}\right) \mid j_{1}^{\ell} H \text { is transversal to } \widetilde{Q}\right\}
$$

is a residual subset of $S p-E m b\left(U, S_{1}^{n}\right)$. If $Q$ is a closed subset, then $T_{Q}$ is open.

We remark that if the corresponding horospherical height function $h_{\mathbf{v}_{0}}$ is $\ell$ - $\mathcal{K}$-determined, then $H$ is a $\mathcal{K}$-versal deformation if and only if $j_{1}^{\ell} H$ is transversal to $\mathcal{K}_{h, \mathbf{v}_{0}}^{\ell}$, where $\mathcal{K}_{h, \mathbf{v}_{0}}^{\ell}$ is the $\mathcal{K}$-orbit through $j^{\ell} h_{\mathbf{v}_{0}}(\mathbf{0}) \in J^{\ell}(n-$ $r, 1)$. Applying Theorem 6.3 , this condition is equivalent to the condition that the corresponding Legendrian immersion germ is Legendrian stable. From the previous arguments and $\S 5$ in [6], we have the following theorem. (See also [1].) 
THEOREM 6.7. If $n \leq 6$, there exists an open subset $\mathcal{O} \subset S p-E m b\left(U, S_{1}^{n}\right)$ such that for any $\mathbf{X} \in \mathcal{O}$, the corresponding Legendrian immersion germ $\mathcal{L}$ is Legendrian stable.

Acknowledgment. The author wishes to express his thanks to Professor Shyuichi Izumiya for useful advices and valuable discussions.

\section{References}

[1] V. I. Arnold, S. M. Gusein-Zade, A. N. Varchenko, Singularities of Differential Maps, Volume I, Birkhäuser, Basel, 1986.

[2] T. Fusho, S. Izumiya, Lightlike surfaces of spacelike curves in de Sitter 3-space, J. Geom. 88 (2008), 19-29.

[3] S. Izumiya, D. Pei, T. Sano, Singularities of hyperbolic Gauss maps, Proc. London Math Soc. 86 (2003), 485-512.

[4] S. Izumiya, M. Kossowski, D. Pei, M. C. Romero Fuster, Singularities of lightlike hypersurfaces in Minkowski four-space, Tohoku Math. J. 58 (2006), 71-88.

[5] S. Izumiya, M. C. Romero Fuster, The lightlike flat geometry on spacelike submanifolds of codimension two in Minkowski space, Sel. Math. NS. 13 (2007), 23-55.

[6] S. Izumiya, D. Pei, M. C. Romero Fuster, M. Takahashi, The horospherical geometry of submanifolds in hyperbolic space, J. London Math. Soc. (2) 71 (2005), 779-800.

[7] M. Kasedou, Singularities of lightcone Gauss images of spacelike hypersurfaces in de Sitter space, J. Geom. 94 (2009), 107-121.

[8] M. Kasedou, Spacelike submanifolds of codimension two in de Sitter space, to appear in J. Geom. Phys. 60 (2010), 31-42.

[9] M. Kasedou, Spacelike submanifolds of codimension at most two in de Sitter space, preprint.

[10] J. A. Montaldi, On contact between submanifolds, Michigan Math. J. 33 (1986), 195199.

[11] G. Wassermann, Stability of Caustics, Math. Ann. 216 (1975), 43-50.

[12] V. M. Zakalyukin, Lagrangian and Legendrian singularities, Funct. Anal. Appl. 10 (1976), 26-36.

[13] V. M. Zakalyukin, Reconstructions of fronts and caustics depending one parameter and versality of mappings, J. Soviet. Math. 27 (1984), 2713-2735.

\section{DEPARTMENT OF MATHEMATICS}

HOKKAIDO UNIVERSITY

SAPPORO 060-0810, JAPAN

E-mail: kasedou@math.sci.hokudai.ac.jp

Received January 14, 2010. 